\title{
Study on Design Symbol of Short Animation Film
}

\author{
Hongjuan Sun \\ School of Art and Design \\ Huanghe Science and Technology College \\ Zhengzhou, China
}

\begin{abstract}
Animation is a category of comprehensive art, and it is an artistic expression form covering various artistic categories such as drawing, cartoon, film, digital media, photography, music and literature. As a media of visual communication, animation is a kind of symbol itself, and it mainly expressed through the aspects of image, color, light and shadow as well as audition.
\end{abstract}

Keywords-symbol; animation image; light and shadow; audition

\section{INTRODUCTION}

The animation image has been evolving with its development since its birth of animation till now. Those familiar animation characters have brought us the unusual relaxation and joyousness, and made the animation more inseparable from our daily life. From the modeling style of animation characters adapting to public tastes with strong publicity to the character modeling of prominent personal style, current animation characters are no longer confined to one expression form.

\section{Symbolic MeAning OF IMAGES IN ANIMATION}

Animation image can be coded using various animation methods, especially the expression techniques such as exaggeration and abstraction, which convey the editor's intention to audiences vividly.

\section{A. Animation Images: Existence of Symbols}

What is symbol? It refers to the logo with certain representative meaning. Generally, it refers to literature, language, code, mathematical symbol, chemical symbol and traffic sign etc. Cassirer, an aesthetician of symbolism, holds the opinion that "art can be defined as a symbolic language", it is the formal symbolic language of our thoughts and emotion. Animation is a special artistic expression form and the character in animated film is a kind of linguistic symbol, conveying the idea of the whole film to audiences through characters' deduction.

In Spanish Altamir Cave paintings, there is a running buffalo with plump and vivid image. What is magical is its tail and legs have been repeatedly painted for many times, forming the sense of movement visually. Similar examples exist in China, as the representative of Majiayao Culture of Neolithic era, there are three groups of dancing figures painted in the "color pottery basin with dancing design".
Each group consists of five dancing figures hands in hands accompanied by singing. Among which, the arms of outer two figures has been repeatedly painted twice to indicate waving arms of the dancing ones. Those are all the original animation intention expression. Along with the development of film technique, animated film has been favored increasingly by the audiences. It is the character in the animated film that first arouses audiences' attention. Then this image is the existence of symbol and animation is a special artistic form of figure symbol. The art style of an animation film has determined the modeling features of this image, and the meaning conveyed to the audiences by this symbol.

Upon the character type of an animation has been confirmed, designers shall design model of characters in detail. The vitality of characters influences the interest expression of whole film directly. While designing the animation characters, generally, attention shall be paid to special visual form, which can stimulate audiences' sense, and be recognized easily, thus easy to be remembered. Characters shall have special modeling elements, and refining and generalization shall be conducted. The animation characters shall be designed adapting to animation requirements starting from plot and summary of character features of the whole film. The selection of character style is of importance in animation creation. Animation characters have various styles, but a successful animation must have a character style that can catch audiences' sight, for example, the villain, hero, and lovable character and so on. Different modeling gives the audiences different mental feelings, and this is the function of symbol of the animation image.

\section{B. Classic Animation Image: Text of Symbol}

The linguistic symbols that are exposed to us in daily life generally appear as the form of text, while animation film conveys information through the performance of animation characters. Animation images are the symbolic text of animation art. For example, when mentioning Disney animation, first of all we will come up with Mickey Mouse, thus the cartoon image of Mickey Mouse is a symbolic text.

The stories of traditional Chinese animation film mainly adapted from Chinese fairy tale with various expression forms, such as ink painting, paper-cut and puppet, as a result, the animation images are of classic Chinese character, and this is the symbolic expression of animation image. Havoc in Heaven is adapted from The Journey to the West, and main 
character in the film is Sun Wukong. The elements of Chinese opera facial designs have adopted for the image design of Sun Wukong, which is a classic representative. And this image has deeply etched in people's mind. "Fig. 1"

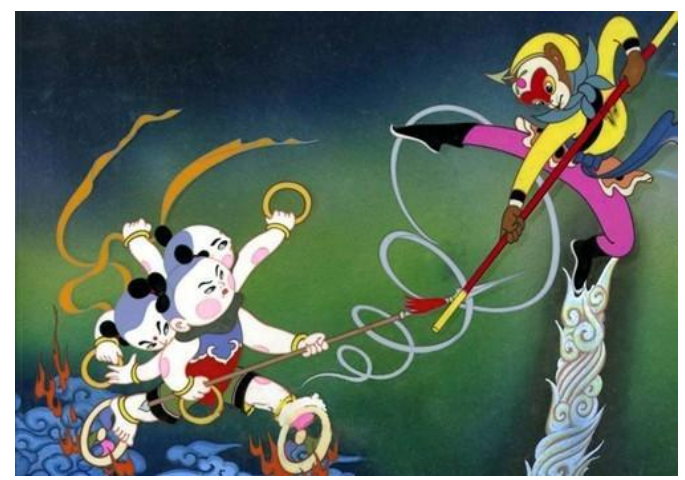

Fig.1 Character Image in the Havoc in Heaven

\section{SYMBOL COMPOSITION OF COLOR IN ANIMATION}

As one of the artistic modeling elements, color is a kind of symbol in terms of semiology. Color may indicate different symbolic meaning in different artistic context because it has been vested symbolism, meaning and emotion. Color is of emotional meaning, as a result, different hue, tone and brightness can bring people different emotional feelings. For example, red stands for enthusiasm, joyousness and positivity; green represents life, youth and environmental protection; black symbolizes death, conceal and dignity; white signifies purity, holy and tidy. Different color may remind people of various matters or scenes in daily life.

Color symbol is the necessary element to form visual cognitive system of animation. In animation film, the design for characters and scenes cannot be done without color. And color is an important part in the artistic design of animation film, rendering the pictures of animation film a strong visual impact. In animation, color refers to leave the audiences some knowledge about certain color, while color symbol signifies the psychological effect that have left to the audiences and the association about color. For example, in the film of Three Monks, little monk wears orange cloths, because orange represents liveliness and youth, which conforms to the age and character of this role. Audiences may feel the features of this role through the color as it is a symbol. In creation of animation film, color symbol can be used to mold different roles; in addition, it can heighten the atmosphere of the whole film. In The Lion King of Disney animation film, the growing environment of Simba is molded as dense tropical rain forest, colorful flowers, and clear waterfall "Fig. 2". However, the living environment of Scar "Fig. 3" is always the dark and gloomy cave. Even though he has obtained the authority to rule all the animals, the thriving prairie becomes a sea of fire. The tone for it is always dark and depressive, and the conveying of picture colors conforms to the plot and atmosphere of this story and through exaggeration of color, atmosphere of the film is heighted.

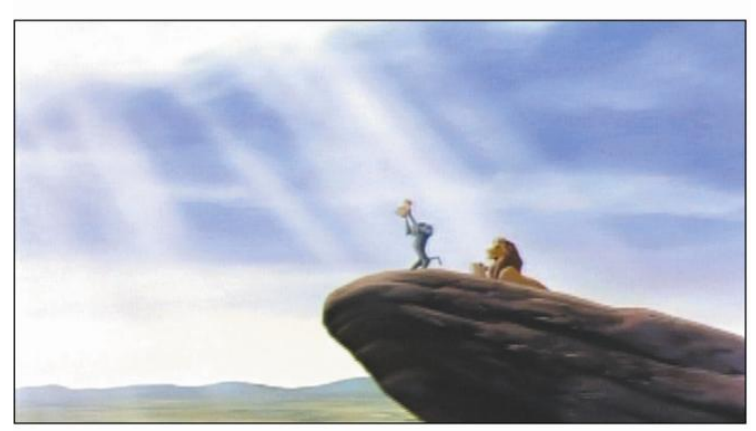

Fig. 2 Scene when Simba was born in The Lion King

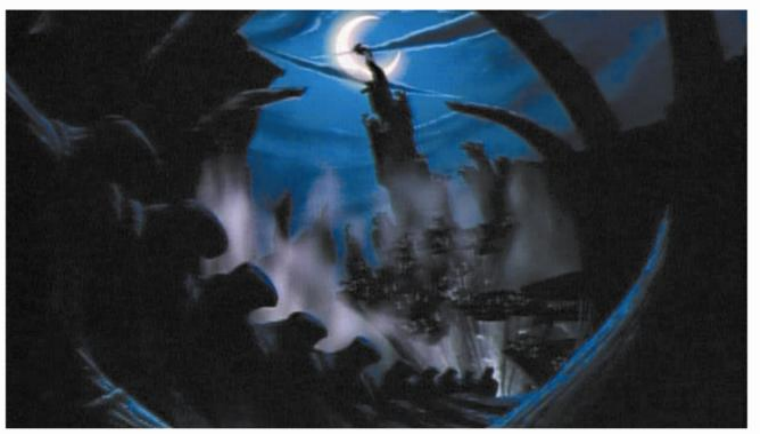

Fig. 3 Elephant graves and ruins in the living environment of Scar in The Lion King

In Addition, color is also playing the role of setoff to express the character's inner feelings and expectations in the animation. In the Spirited Away of Hayao Miyazaki's animation works, the scene for Chihiro and her parents entering the tunnel is the dark and long tunnel, indicating Chihiro's fear about tunnel. After walking a long time in the tunnel, there is a little light at the end, reflecting Chihiro's desire to walk out the tunnel quickly to see the scene at the other end of the tunnel.

The application of color in different theme and different nation has distinctive different style as well. Disney animation takes fairy tale as its main theme, so the color style of Disney animation is mostly brilliant, bright, lovable; influenced by traditional Chinese art, the color of traditional Chinese animation film has a strong contrast, possessing the unique features of traditional Chinese painting. The animation works of Hayao Miyazaki mainly express the theme of environmental protection, vitality, dream and life. So the film colors are brilliant and pure, with main color of the picture as cyan. And most of the scenes are clear water flow, and flourishing forest.

The color symbol is an important element to form the animation. The author expresses his/her creative intention through color matching and tone comparison. The picture conveys thought to audiences via color besides modeling.

\section{EXPRESSION OF LIGHT AND SHADOW IN THE ANIMATION}

Light can be divided into natural light and artificial light. Natural light mainly refers to the direct radiation of sunlight 
or the lights formed by reflection or refraction of sunlight. Artificial light refers to the man-made light source in indoor environment.

Objects radiated by light source may generate shadows, and the shadow may be a different one from various perspective.

Thanks to the radiation of light, we can see colorful world and feel the charming environment. Therefore, we will see nothing without light. Light may contribute to the expression of material texture; in addition, light may also construct the feeling of cold and warm, false and true, strong and weak. Shadow is of metaphor and symbolic meaning itself, rendering us the feeling of mysterious in terms of visual. In animation film, light and shadow is of vital importance.

Without light, there is no shadow. Light and shadow just like a twin. As a symbol, light and shadow has not only shaped the feeling of space as for the scene, but also implied the mental feelings of the audiences "Fig. 4".
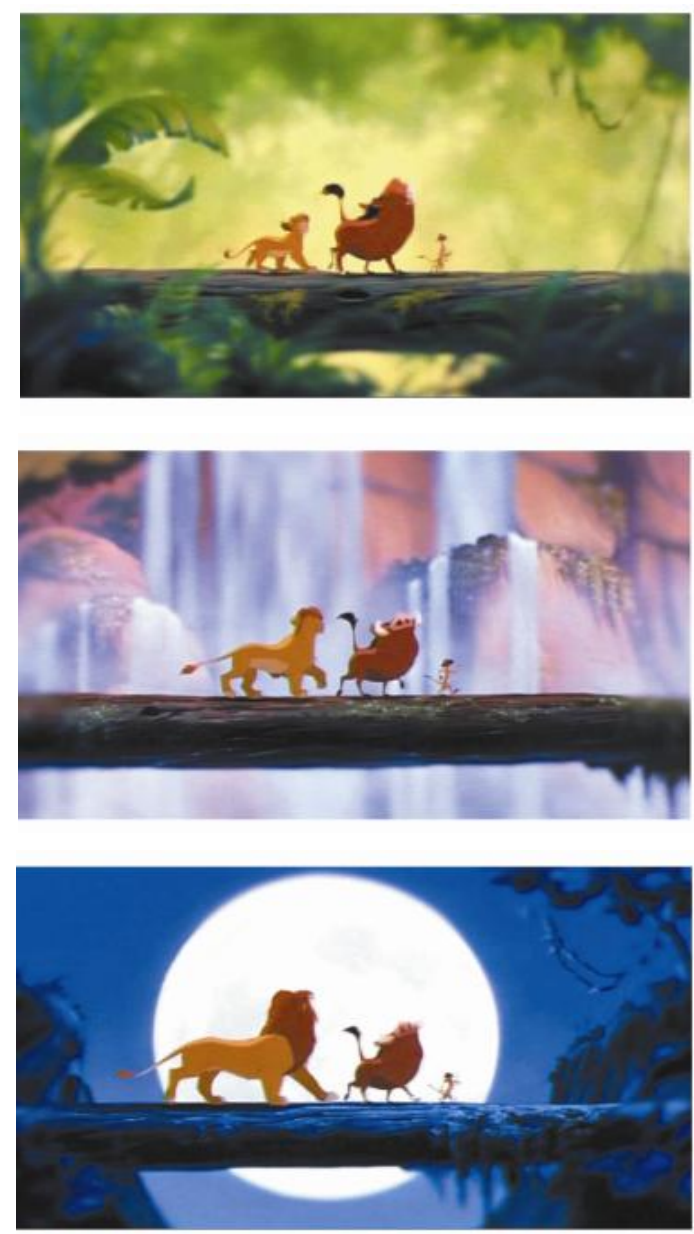

Fig. 4 Different Light and Shadow Effect in the Same Scene of The Lion King

\section{FUNCTION OF THE AUDITORY SYMBOL IN ANIMATION}

The information in animation is conveyed through moving pictures accompanied by sounds. Audio-visual language is an exclusive category of art for the film; meanwhile, it is a symbolic coding system in the mass media. Animation film belongs to a category of film. "Hearing" is an important element in the audio-visual language. The reason for an attractive animation film is the enjoyment in terms of auditory it has vested to the audiences besides the charming pictures. Therefore, as an expression of symbol, audio-visual language is of its own unique function.

Lange holds the opinion that language is one of most advanced and appalling symbols created by human till now. As for human's hearing, language and sounds, which are the auditory memory conveyed by an animated character to the audiences, are easily to be remembered by human brain. From the perspective of semiology, the auditory symbols signifies various human voices, sounds and music in real life, and it refers to the mental and physical feelings brought to audiences by sounds, making the pictures of animation film more vivid, and bring audiences into the plot of story easier.

Auditory symbol is of sense of rhythm and appeal, and sounds can be used to sum up theme, render atmosphere, and stress the film style in the animation film. Sounds is expressed through auditory images, for example the characters' sounds, including their dialogue and monologue. The sound effect and background music generated from the environment tell the audiences the theme of this film.

Besides its function of explaining the pictures, sounds is also the element of one play. Screen images and specific personal character in films are always shaped and depicted via sounds. Sounds may convey the contents beyond picture to the audiences, strengthening the interactive rhythm of sound-picture. Different combination of form and content between sounds and pictures may create various effects with expressive force, deepening the content and meaning of the picture. The success of Three Monks is not only for its unique artistic style, but also due to the success of its music and sound. There is no dialogue and voiceover in the whole film and the music color is enriched by the unique Chinese percussion instruments such as drum and cymbal. Music has played an important role both in depicting personal character, rendering emotions and environmental atmosphere, and promoting the plot development. Use different instruments to depict different roles, stressing personal character via the features of instrument itself. Take advantage of the clear and melodious sound of Banhu to depict the smart and lively character of little monk; the mean tall monk is described via the flexible Zhuihu; the fat monk is depicted using Guanzi whose tone is rich, obtaining the artistic effect of "doing more with less", strengthening the enjoyment and richness of the whole film.

Each person has his own speaking habits and language style, and animated characters are no exception. For example, the classic dialogue in Japanese animation film of Detective Conan is "There is only one truth", reminding people of the role of Conan who looks like being shrunken by wearing a big glass. Only by large quantity of repetition, can the linguistic symbol be accepted and remembered by the audiences. However, repeating blindly is infeasible; it shall be used in specific occasion. In addition to the language of 
character, the theme song of the film is of same function. Love Me Tonight, the famous song in The Lion King, is playing the same function. The love story of Simba and Nana will come into people's mind when they heard this song.

Sound plays a vital role in animation film; it can grasp the pace of film, render atmosphere, express the character's inner world, and show the country's cultural connotation. In modern animation works, sound not only plays the role of setting off pictures of the film, but also it has broke away from the subsidiary position of picture as an independent symbol, possessing the aesthetic value and research meaning of its own.

\section{CONCLUSION}

In conclusion, animation design symbol must have the following three conditions: first, the various visual elements expressed in the animation, namely the animation character image; second, the animation film itself; third, channels of communication, namely such media as cinema or television, through which, audiences may learn the deep meaning of animation. In addition to make the audiences feel the image, color, light and shadow, as well as audition of the animation film, animation symbol also express the symbolic abstract information. Animation is accepting more and more acknowledgement form audiences via its unique expression form of character modeling and aesthetic value. In the context of popular fashion and popular culture, and as a cultural awareness symbol, animation makes audiences to connect it with the symbol in their memory quickly through transmitting and outputting, arousing emotional and psychological resonance, and subtly influence the change of aesthetic awareness, aesthetic taste and aesthetic value, making it a new aesthetic form and aesthetic value outlook.

\section{REFERENCES}

[1] Jia Fou, Lu Shengzhang. Introduction to Animation [M]. Communication University of China Press, 2005.

[2] Wang Huaqing, Min Xiaoyun. Symbolic Language in Animation Image Design [J]. Film Literature, 2009, 17.

[3] Gao Yongli. Animation Art in Symbol Meanings [J]. Scientific and Technological Information (Scientific Teaching and Research), 2007, 07. 